\title{
In diesem Heft
}

\author{
125 Editorial \\ 128 Diskussion \\ 129 Volker Bach \\ Grußwort

\section{Notizen} \\ I30 Kristina Vaillant im Gespräch mit Sebastian Telsemeyer \\ Mathe studiert - und dann? \\ 132 Leoni Winschermann \\ Mathematikstudium mal anders \\ 136 Florian $\mathrm{Heß}$ und Gregor Kemper \\ Fachgruppe Computeralgebra: 29-jähriges Bestehen
}

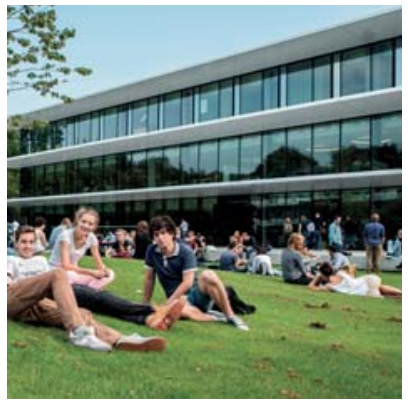

\section{Aktuell}

138 Antje Schulz

News, Tipps und Termine

I40 Klaus Hulek

zbMATH - Gedanken zur Zukunft

\section{Fokus}

I44 Gustav Holzegel

Gravitationswellen

\section{Publikum}

153 Kitty Hawk

Wir sollten es positiv sehen

I54 Sylvie Paycha

Blumen, Gemüse und Mathematik - Porträts und Interviews mit dreize hn Mathematikerinnen

\section{Panorama}

157 Georg Wilhelm Scheffers

Curiosa I: Fragen, die meist falsch beantwortet werden

160 Dirk Holzberg

Naturkonstruktionen

163 Michael Joswig

Leibniz und der Garten

167 Thilo Kuessner

Logbuch Mathematik 


\section{Lehren und Lernen}

Franz Lemmermeyer

Abituraufgaben und Kompetenz

\section{Retrospektive}

I74 Benedikt Löwe

Die Hamburger DMV-Jahresversammlungen I90I und I928

\section{Rätsel}

Brigitte Lutz-Westphal

CD-Durcheinander: Die Seite für Kinder

\section{DMV intern}

181 Ergebnis der Präsidiumswahlen

I8I DMV-Ansprechpartner/innen vor Ort

181 Die DMV

Personalia

182 Informationen

I82 Neue Mitglieder und Todesfälle

I82 Berufungen, Habilitationen und Promotionen

I84 Impressum 